\title{
A Novel Conformable Fractional Nonlinear Grey Bernoulli Model and Its Application
}

\author{
Wanli Xie ${ }^{1}$ and Guixian Yu ${ }^{2}$ \\ ${ }^{1}$ Institute of EduInfo Science and Engineering, Nanjing Normal University, Nanjing, Jiangsu 210097, China \\ ${ }^{2}$ School of Software \& Microelectronics, Peking University, Beijing 100741, China \\ Correspondence should be addressed to Guixian Yu; yuguixian@pku.edu.cn
}

Received 29 May 2020; Revised 10 August 2020; Accepted 3 September 2020; Published 19 September 2020

Academic Editor: Hassan Zargarzadeh

Copyright ( 2020 Wanli Xie and Guixian Yu. This is an open access article distributed under the Creative Commons Attribution License, which permits unrestricted use, distribution, and reproduction in any medium, provided the original work is properly cited.

\begin{abstract}
The fractional nonlinear grey Bernoulli model, abbreviated as $\operatorname{FANGBM}(1,1)$, is a successful extension of NGBM $(1,1)$. Although FANGBM $(1,1)$ has numerous excellent characteristics, it has a more complex form of fractional accumulation (FA) operator than raw $\operatorname{NGBM}(1,1)$. In this study, we propose a novel fractional nonlinear grey Bernoulli model, named CFNGBM $(1,1)$, which uses conformable fractional accumulation (CFA), which has a simpler form than FANGBM. Using two practical cases, the effectiveness of the proposed CFNGBM $(1,1)$ in practical applications was illustrated. Results show that the CFNGBM $(1,1)$ exhibited higher accuracy than other grey models, thus facilitating its promotion in engineering practices.
\end{abstract}

\section{Introduction}

Deng first proposed the grey system theory, which significantly advanced the development of systems science [1]. As a crucial branch of the grey system theory, grey forecasting models have become a research hotspot for scholars. The grey forecasting model is based on data that have some known and unknown information in the real world. Nowadays, this model is widely used in industrial, economic, energy, agricultural, and other fields [2-9]. $\operatorname{GM}(1,1)$ is the core model in the grey forecasting models. Moreover, it has a good modeling effect on sequences with exponential law and can reveal future development trends of the system [2-9]. The GM $(1,1)$ modeling process accumulates the original sequence to obtain a first-order accumulative generating operator (1-AGO) sequence, which can weaken the randomness of the original data and fully expose the information contained in the original sequence $[9,10]$. Recently, many researchers have conducted a series of fruitful work to further improve the modeling accuracy of the grey models. Considering the varying spans of differential equations, Xie and Liu [10] constructed the DGM $(1,1)$ model and analyzed its unbiased characteristics based on the perspective of discrete to discrete modeling. Cui et al. [11] reported that the grey model based on $\mathrm{GM}(1,1)$ can well fit the data series with pure exponential law characteristics; however, in reality, a large amount of data exists with nonhomogeneous exponential characteristics in addition to homogeneous exponential data. To further expand the applicability of the grey model, Cui et al. [11] proposed $\operatorname{NGM}(1,1, k)$, which can simulate sequences with nonhomogeneous exponential law. Zeng et al. [12] proposed a novel self-adapting intelligent grey model and used it to predict natural gas demand. Furthermore, the grey Verhulst model can better fit sequences with saturated growth or unimodal characteristics [4]. Recently, Wu et al. [13] proposed the fractional accumulation and fractional difference to improve further grey models, and this method was proved to overcome the drawback by the integer-order accumulation. Subsequently, some novel fractional grey models were proposed gradually. For instance, Yang and Xue [14] investigated the concept of fractional derivative and then used the G-L fractional derivative to deduce a new grey prediction model, and this model was applied to China's electricity prediction. Zhu et al. [15] suggested a new grey model to forecast Jiangsu's electricity consumption in China, and the results showed 
that the proposed model had higher accuracy than other models.

Based on the above analysis, these models are mostly noted to be linear models or only suitable for a specific type of data. To characterize the complex laws of the system in the real world, the model structure must be flexibly adjusted according to the actual background of the modeling and should describe the nonlinear characteristics of data. Thus, to achieve these objectives, the nonlinear grey Bernoulli model (NGBM) was proposed [16]. The NGBM is a firstorder univariate grey system model that can be used to describe the nonlinear development of data. The main advantage of the $\operatorname{NGBM}(1,1)$ is that the power index in the grey interaction can better reflect the nonlinear characteristics of the original data [16]. Furthermore, particle swarm optimization and genetic algorithm were used to optimize the power index in the $\operatorname{NGBM}(1,1)$ and achieve good forecast results [17, 18]. Wang et al. [19] established a nonlinear optimization model to simultaneously optimize the background value and power index, further enhancing the predictive ability of the model; this optimization model was used to facilitate early warning of the attainment rate of industrial wastewater in 31 administrative regions in China. Liu and Xie [20] proposed a novel nonlinear grey model, namely, $\operatorname{WBGM}(1,1)$, which was based on the Weibull cumulative distribution function, in which the nonlinear parameter was optimized by the genetic algorithm, and thereby, many valuable results were found. Although the grey Bernoulli model has numerous applications in reality and solves many difficult problems, it heavily relies on the selection of parameters. Although many existing methods have been proposed for optimizing the parameters, the order number of these models is an integer. The order of integer may prevent the model from achieving the best effect because the appropriate order must be selected based on actual data. Wu et al. [21] reported a new modeling method of fractional nonlinear grey Bernoulli model, called FANGBM $(1,1)$. This model was successfully applied to China's short-term energy consumption forecasting. However, the fractional accumulation (FA) of $\operatorname{FANGBM}(1,1)$ has more complicated mathematical expressions. Moreover, its implementation in programming is challenging when the mathematical foundation of engineers is relatively weak. To better extend the NGBM to engineering practices, herein, we present a novel fractional nonlinear grey Bernoulli model, named CFNGBM $(1,1)$, in which the construction of FA is easily extended to engineering. To verify the effectiveness of the novel model, the model is verified by the case of carbon dioxide emissions in China.

The emission of carbon dioxide directly affects ecosystem as well as the sustainable economic development. The reliable projection of $\mathrm{CO}_{2}$ emissions can assist decisionmakers in shaping the associated energy policy. For this, many scholars have carried out a lot of effective works [22-24]. Due to the rapid development of China's economy, carbon dioxide emissions are inevitably affected by economic policies, with dynamic changes. In accordance with the previous literature, the grey Bernoulli model with conformable fractional accumulation is developed for
China's carbon dioxide forecasting, in which the nonlinear parameter of the novel model is determined by a bioinspired algorithm, namely, the whale optimization algorithm (denoted as WOA for short).

On these theoretical bases, we summarize the contributions of our work as follows:

(1) A case is presented to demonstrate that FA and CFA are close in value

(2) The novel fractional nonlinear grey Bernoulli model, $\operatorname{CFNGBM}(1,1)$, is discussed

(3) The parameter estimation and optimization methods of the order and power index of the CFNGBM $(1,1)$ are provided

(4) Two empirical cases are used to verify the accuracy of the proposed model

The remaining of this paper is organized as follows. First, Section 2 provides an introduction to the previous fractional nonlinear grey Bernoulli model. Next, the proposed grey model is presented in Section 3. Section 4 presents the optimization method of our model. Section 5 demonstrates the verification method of our model. Finally, Section 6 summarizes the conclusions of this study.

\section{Description of the FANGBM $(1,1)$}

In this section, we provide a brief review of the FANGBM $(1,1)$. Wu et al. [21] proposed the fractional nonlinear grey Bernoulli model and successfully applied it to an engineering practice. The $\operatorname{FANGBM}(1,1)$ is defined as follows:

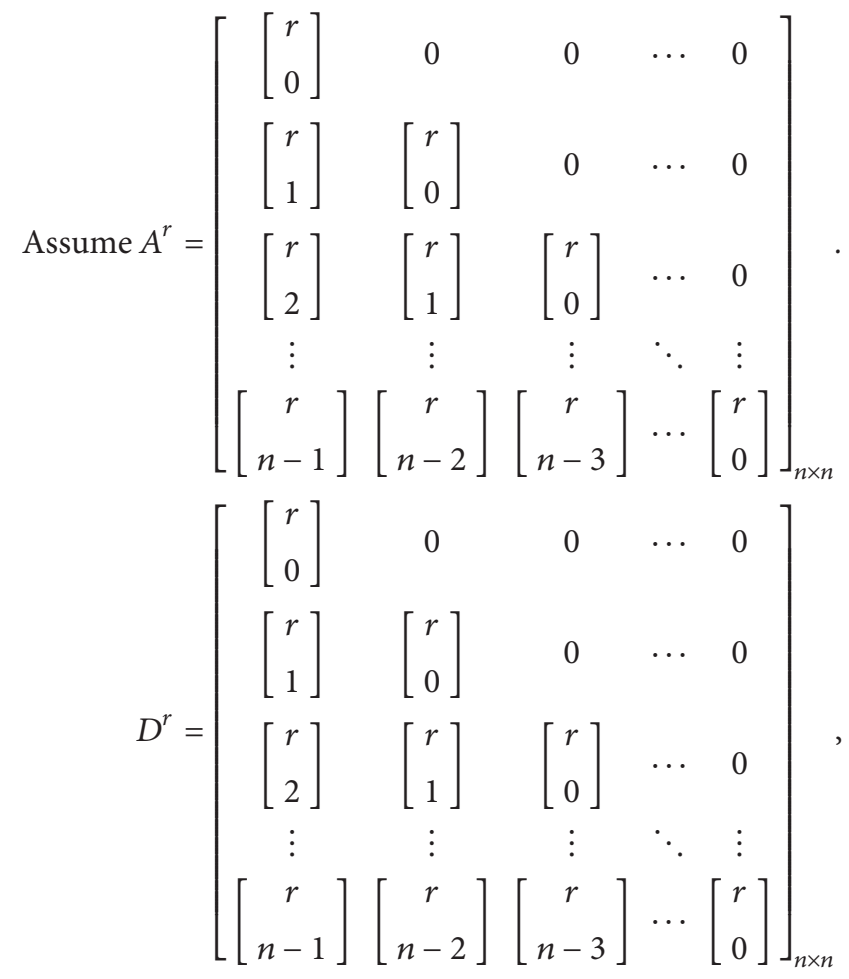


with

$$
\begin{aligned}
& {\left[\begin{array}{l}
r \\
i
\end{array}\right]=\frac{(r+i-1) !}{i !(r-1) !}} \\
& {\left[\begin{array}{l}
0 \\
i
\end{array}\right]=0,} \\
& {\left[\begin{array}{l}
0 \\
0
\end{array}\right]=1, \quad D^{r} \text { 中 } i>r .}
\end{aligned}
$$

Assume

$$
X^{(0)}=\left\{x^{(0)}(1), x^{(0)}(2), \ldots, x^{(0)}(n)\right\},
$$

to be the nonnegative series; then, the $r$-order accumulative generating operator (r-AGO) series [16] is

$$
X^{(r)}=\left\{x^{(r)}(1), x^{(r)}(2), \ldots, x^{(r)}(n)\right\},
$$

where $X^{(r)}=\mathbf{A} r X^{(0)}$. The equation

$$
\frac{\mathrm{d} x^{(r)}(t)}{\mathrm{d} t}+a x^{(r)}(t)=b x^{(r)}(t)^{\gamma}
$$

is called the whitening equation of $\operatorname{FANGBM}(1,1)$. Here, to obtain the structural parameters of $\operatorname{FANGBM}(1,1)$, the discrete form of equation (5) should be deduced. For this, a crucial result (Ref. [8]) is introduced to discretize equation (5). Let $g_{\omega}$ be the mapping function, and if $g_{\omega}$ is a grey mapping function, it should satisfy the following:

(i) $g_{\omega}: S\left(\omega_{i}\right) \longrightarrow S\left(g_{i}\right)$,

(ii) $g_{1}: \mathrm{d} x^{(r)}(t) / \mathrm{d} t \longrightarrow x^{(r-1)}(k)=x^{(r)}(k)-x^{(r)}(k-$

1), $g_{2}: x^{(r)}(t) \longrightarrow z^{(r)}(k)$, (iii) $\left(g_{1}, g_{2}\right)$ is the basic mapping of whitening differential equations,

where $S\left(g_{i}\right), i=1,2, \ldots$, is the grey item set and $g_{i}$ is the grey item mapping, $S\left(\omega_{i}\right), i=1,2, \ldots$ is the white item set, and $\omega_{i}$ is the white item mapping.

By the basic mapping of the whitening differential equation, equation (5) becomes

$$
x^{(r)}(k)-x^{(r)}(k-1)+a z^{(r)}(k)=b z^{(r)}(k)^{\gamma},
$$

where $z^{(r)}(k)$ denotes the background value and is obtained as

$$
z^{(r)}(k)=0.5\left(x^{(r)}(k)+x^{(r)}(k-1)\right) .
$$

Therefore, after using the least-squares method to estimate the parameters $(a, b)^{T}$, the solution of equation (5) with $x^{(r)}(1)=x^{(0)}(1)$ becomes

$$
\widehat{x}^{(r)}(k)=\left(\left(x^{(0)}(1)^{(1-\gamma)}-\left(\frac{b}{a}\right)\right) e^{-a(1-\gamma)(k-1)}+\left(\frac{b}{a}\right)\right)^{(1 / 1-\gamma)} .
$$

Using the $r$-order inverse AGO ( $r$-IAGO), the simulated values of $X^{(0)}$ are solved as follows:

$$
X^{(0)}=\mathbf{A}^{(-r)} \widehat{X}^{(r)} .
$$

\section{Methodology}

3.1. Conformable Fractional Accumulation and Difference. In this subsection, we briefly review conformable fractional accumulation (CFA) and conformable fractional difference (CFD). Ma et al. [25] presented the following definitions of CFA and CFD:

$$
\begin{aligned}
& \nabla^{r} f(k)=\left\{\begin{array}{l}
\nabla\left(k^{r-1} f(k)\right)=\sum_{i=1}^{k} \frac{f(i)}{i^{1-r}}, \quad r \in(0,1], k \in N^{+}, \\
\nabla^{(n+1)}\left(k^{r-[r]} f(k)\right), \quad r \in(n, n+1], k \in N^{+} .
\end{array}\right. \\
& \Delta^{r} f(k)= \begin{cases}k^{1-r} \Delta f(k)=k^{1-r}[f(k)-f(k-1)], \quad r \in(0,1], k \in N^{+}, \\
k^{[r]-r} \Delta^{n+1} f(k), & r \in(n, n+1], k \in N^{+} .\end{cases}
\end{aligned}
$$


Thus, the CFA operator has a simpler form than the CFD operator. In particular, when $r \in(0,1]$, it has the following form:

$$
\begin{aligned}
& x_{(r)}(k)=\sum_{i=1}^{k} \frac{x^{(0)}(i)}{i^{1-\alpha}} x^{(0)}(i)=\left[x^{(0)}(1), x^{(0)}(2), \ldots, x^{(0)}(n)\right]\left(\begin{array}{ccccc}
1 & 1 & \cdots & 1 & 1 \\
& \frac{1}{2^{1-\alpha}} & \cdots & \frac{1}{2^{1-\alpha}} & \frac{1}{2^{1-\alpha}} \\
\vdots & \vdots & \vdots & \vdots & \vdots \\
0 & 0 & \cdots & \frac{1}{(n-1)^{1-\alpha}} \frac{1}{(n-1)^{1-\alpha}} \\
& & & & \frac{1}{n^{1-\alpha}}
\end{array}\right) \text {. } \\
& =\left[x^{(0)}(1), x^{(0)}(2), \ldots, x^{(0)}(n)\right]\left(\begin{array}{ccccccc}
1 & 0 & \cdots & 0 & & 0 \\
& & 1 & & & & \\
0 & \frac{1}{2^{1-\alpha}} & \cdots & 0 & & 0 \\
\vdots & \vdots & \vdots & \vdots & \vdots \\
0 & 0 & \cdots & \frac{1}{(n-1)^{1-\alpha}} & 0 \\
& & & & & & \\
0 & 0 & \cdots & 0 & \frac{1}{n^{1-\alpha}}
\end{array}\right)\left(\begin{array}{cccccc}
1 & 1 & \cdots & 1 & 1 \\
0 & 1 & \cdots & 1 & 1 \\
\vdots & \vdots & \vdots & \vdots & \vdots \\
0 & 0 & \cdots & 1 & 1 \\
0 & 0 & \cdots & 0 & 1
\end{array}\right) \text {. } \\
& x^{(r)}(2)-x^{(r)}(1)+a z^{(r)}(2)=b\left(z^{(r)}(2)\right)^{\gamma} \text {, } \\
& x^{(r)}(3)-x^{(r)}(2)+a z^{(r)}(2)=b\left(z^{(r)}(3)\right)^{\gamma} \text {, } \\
& x^{(r)}(n)-x^{(r)}(n-1)+a z^{(r)}(n)=b\left(z^{(r)}(n)\right)^{\gamma} .
\end{aligned}
$$
operators, the sequence $[1,2,1.5,1.3]$ provided in the litvalue with different orders (Table 1). Moreover, the mean and standard deviations are quite similar, which shows that CFA can be a good substitute for FA.

3.2. The Proposed CFNGBM $(1,1)$ Model. In this subsection, we provide the definition of the $\operatorname{CFNGBM}(1,1)$. Let the original sequence be $X^{(0)}=\left(x^{(0)}(1), x^{(0)}(2), \ldots, x^{(0)}(n)\right)$; thus, its $r$-order CFA is $X^{(r)}=\left(x^{(r)}(1), x^{(r)}(2)\right.$, $\left.\ldots, x^{(r)}(n)\right)$, where

$$
x^{(\alpha)}(k)=\nabla^{\alpha} x^{(0)}(k)= \begin{cases}\sum_{j=1}^{k} \frac{x^{(0)}(j)}{j^{[\alpha]-\alpha}}, & 0<\alpha \leq 1 . \\ \sum_{j=1}^{k} x^{(\alpha-1)}(j), & \alpha>1 .\end{cases}
$$

The $\operatorname{CFNGBM}(1,1)$ is

$$
x^{(r)}(k)-x^{(r)}(k-1)+a z^{(r)}(k)=b\left(z^{(r)}(k)\right)^{\gamma} .
$$

By substituting the data into the $\operatorname{CFNGBM}(1,1)$, we achieve
Within discrete form (13), we can easily get the parameter of the $\operatorname{CFNGBM}(1,1)$ using the least-squares method with given samples (14) as

$$
\theta=[\widehat{a}, \widehat{b}]^{T}=\left(B^{T} B\right)^{-1} B^{T} Y,
$$

where

$$
\begin{aligned}
& B=\left[\begin{array}{cc}
-z^{(r)}(2) & \left(z^{(r)}(2)\right)^{\gamma} \\
-z^{(r)}(3) & \left(z^{(r)}(3)\right)^{\gamma} \\
\vdots & \vdots \\
-z^{(r)}(v) & \left(z^{(r)}(v)\right)^{\gamma}
\end{array}\right], \\
& Y=\left[x^{(r-1)}(2), x^{(r-1)}(3), \ldots, x^{(r-1)}(n)\right]^{T} .
\end{aligned}
$$

We use $-\widehat{a} z^{(r)}(k)+\widehat{b}\left(z^{(r)}(k)\right)^{\gamma}$ instead of $x^{(r)}(k)-x^{(r)}(k-1), k=2,3, \ldots, n$. Thus, we achieve the error sequence $\varepsilon=Y-B \theta$, where $Y, B$, and $\theta$ are defined in equation (15). Set 
TABLE 1: Comparison of FA and CFA with different orders.

\begin{tabular}{lcccccccccc}
\hline Raw data & \multicolumn{3}{c}{ FA } & & & & \multicolumn{3}{c}{ CFA } \\
Value & $0=0.2$ & $r=0.6$ & $r=0.1$ & $r=1.4$ & $r=1.8$ & $r=0.2$ & $r=0.6$ & $r=0.1$ & $r=1.4$ & $r=1.8$ \\
\hline 1.0 & 1.0 & 1.0 & 1.0 & 1.0 & 1.0 & 1.0 & 1.0 & 1.0 & 1.0 \\
2 & 2.2 & 2.6 & 3.0 & 3.4 & 3.8 & 2.1 & 2.5 & 3.0 & 3.3 & 3.7 \\
1.5 & 2.0 & 3.1 & 4.5 & 5.9 & 7.6 & 2.7 & 3.4 & 4.5 & 6.4 & 7.6 \\
1.3 & 3.6 & 5.2 & 7.5 & 10.3 & 13.9 & 3.7 & 5.2 & 7.5 & 10.8 & 13.9 \\
Average & 2.2 & 2.9 & 4 & 5.1 & 6.5 & 2.3 & 3.0 & 4 & 5.3 & 6.5 \\
Standard & 1.0 & 1.7 & 2.7 & 3.9 & 5.5 & 1.1 & 1.7 & 2.7 & 4.2 \\
\hline
\end{tabular}

$s=\varepsilon \cdot \varepsilon^{T}=\sum_{k=2}^{n}\left(x^{(r)}(k)-x^{(r)}(k-1)+\widehat{a} z^{(r)}(k)-\widehat{b}\left(z^{(r)}(k)\right)^{\gamma}\right)^{2}$.
To minimize $s, \widehat{a}$ and $\widehat{b}$ should satisfy

$$
\left\{\begin{array}{l}
\left(\frac{\partial s}{\partial \widehat{a}}\right)=2 \sum_{k=2}^{n}\left(x^{(r)}(k)-x^{(r)}(k-1)+\widehat{a} z^{(r)}(k)-\widehat{b}\left(z^{(r)}(k)\right)^{\gamma}\right) z^{(r)}(k)=0 \\
\left(\frac{\partial s}{\partial \widehat{b}}\right)=-2 \sum_{k=2}^{n}\left(z^{(r)}(k)\right)^{\gamma}\left(x^{(r)}(k)-x^{(r)}(k-1)+\widehat{a} z^{(r)}(k)-\widehat{b}\left(z^{(r)}(k)\right)^{\gamma}\right)=0 .
\end{array}\right.
$$

Theorem 1. We set

$$
\begin{aligned}
& D=\sum_{k=2}^{n}\left[z^{(1)}(k)\right]^{2} . \\
& E=\sum_{k=2}^{n}-\left[z^{(1)}(k)\right]^{(\gamma+1)} . \\
& G=\sum_{k=2}^{n}-\left[z^{(1)}(k)\right]^{(\gamma+1)} . \\
& H=\sum_{k=2}^{n}\left[z^{(1)}(k)\right]^{2 \gamma} . \\
& J=-\sum_{k=2}^{n} z^{(r)}(k) x^{(r-1)}(k) . \\
& L=\sum_{k=2}^{n}\left(z^{(r)}(k)\right)^{\gamma} x^{(r-1)}(k) .
\end{aligned}
$$

$$
\begin{aligned}
& B^{T} B=\left[\begin{array}{cccc}
-z^{(r)}(2) & -z^{(r)}(3) & \cdots & -z^{(r)}(n) \\
\left(z^{(r)}(2)\right)^{\gamma} & \left(z^{(r)}(3)\right)^{\gamma} & \cdots & \left(z^{(r)}(n)\right)^{\gamma}
\end{array}\right] \\
& {\left[\begin{array}{cc}
-z^{(r)}(2) & \left(z^{(r)}(2)\right)^{\gamma} \\
-z^{(r)}(3) & \left(z^{(r)}(3)\right)^{\gamma} \\
\vdots & \vdots \\
-z^{(r)}(n) & \left(z^{(r)}(n)\right)^{\gamma}
\end{array}\right]} \\
& =\left[\begin{array}{cc}
\sum_{k=2}^{n}\left[z^{(1)}(k)\right]^{2} & \sum_{k=2}^{n}-\left[z^{(1)}(k)\right]^{\gamma+1} \\
\sum_{k=2}^{n}-\left[z^{(1)}(k)\right]^{\gamma+1} & \sum_{k=2}^{n}\left[z^{(1)}(k)\right]^{2 \gamma}
\end{array}\right]=\left[\begin{array}{cc}
D & E \\
G & H
\end{array}\right] .
\end{aligned}
$$

When $\left|B^{T} B\right| \neq 0$, the parameters of the model also can be obtained using the following method, $\theta=[\widehat{a}, \widehat{b}]^{T}$, where

$$
\theta=\left(\frac{1}{\mathrm{DH}-\mathrm{GE}}\right)\left[\begin{array}{l}
\mathrm{HJ}-\mathrm{EL} \\
\mathrm{DL}-\mathrm{GJ}
\end{array}\right]
$$

Then,

$$
\begin{aligned}
\left|B^{T} B\right| & =\mathrm{DH}-\mathrm{GE} . \\
\left(B^{T} B\right)^{*} & =\left[\begin{array}{cc}
H & -E \\
-G & D
\end{array}\right], \quad \text { if }\left|B^{T} B\right| \neq 0 .
\end{aligned}
$$




$$
\begin{aligned}
& \left(B^{T} B\right)^{-1}=\left(\frac{1}{\left|B^{T} B\right|}\right)\left(B^{T} B\right)^{*}=\left(\frac{1}{\mathrm{DH}-\mathrm{GE}}\right)\left[\begin{array}{cc}
H & -E \\
-G & D
\end{array}\right], \\
& B^{T} Y=\left[\begin{array}{cc}
-z^{(r)}(2) & \left(z^{(r)}(2)\right)^{\gamma} \\
-z^{(r)}(3) & \left(z^{(r)}(3)\right)^{\gamma} \\
\vdots & \vdots \\
-z^{(r)}(n) & \left(z^{(r)}(n)\right)^{\gamma}
\end{array}\right]^{T}\left[\begin{array}{c}
x^{(r)}(2)-x^{r}(1) \\
x^{(r)}(3)-x^{r}(2) \\
\vdots \\
x^{(r)}(v)-x^{r}(v-1)
\end{array}\right] \\
& =\left[\begin{array}{c}
-\sum_{k=2}^{n} z^{(r)}(k) x^{(r-1)}(k) \\
\sum_{k=2}^{n}\left(z^{(r)}(k)\right)^{\gamma} x^{(r-1)}(k)
\end{array}\right]=\left[\begin{array}{l}
J \\
L
\end{array}\right] .
\end{aligned}
$$

The parameters of the model can be obtained using the least-squares method:

$$
\begin{aligned}
\theta & =[\hat{a}, \widehat{b}]^{T}=\left(B^{T} B\right)^{-1} B^{T} Y=\left(B^{T} B\right)^{-1}\left(B^{T} Y\right) \\
& =\frac{1}{\mathrm{DH}-\mathrm{GE}}\left[\begin{array}{c}
\mathrm{HJ}-\mathrm{EL} \\
\mathrm{DL}-\mathrm{GJ}
\end{array}\right] .
\end{aligned}
$$

The whitening equation of the $\operatorname{CFNGBM}(1,1)$ is

$$
\frac{\mathrm{d} x^{(r)}(t)}{\mathrm{d} t}+a x^{(r)}(t)=b\left(x^{(r)}(t)\right)^{\gamma}, \quad r>0 .
$$

The time response function of the $\operatorname{CFNGBM}(1,1)$ with $\theta=[\widehat{a}, \widehat{b}]^{T}$ is expressed as

$$
\begin{array}{r}
\widehat{x}^{(r)}(k)=\left[\left(\left(x^{(r)}(1)\right)^{(1-\gamma)}-\left(\frac{\widehat{b}}{\hat{a}}\right)\right) e^{-a(1-\gamma)(k-1)}+\left(\frac{\widehat{b}}{\hat{a}}\right)\right]^{(1 / 1-\gamma)}, \\
k=2,3, \ldots, n .
\end{array}
$$
follows:

The predicted value of the model can be obtained as

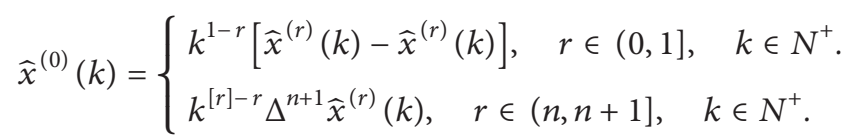

Therefore, we can observe that $\operatorname{CFNGBM}(1,1)$ has a simpler structure than $\operatorname{FANGBM}(1,1)$.

\section{Parameter Optimization of the CFNGBM(1,1)}

In the above descriptions, order $r$ and power index $\gamma$ are assumed to be known; however, they are often changeable in a different system that requires flexible adjusting over a given modeling background. To optimize the parameters of the model, we used the whale optimization algorithm (WOA), proposed by Mirjalili and Lewis [27] to automatically determine the order and power index. According to the hunting behavior of humpback whales, this intelligent algorithm lets the current best candidate solution (agent) be the target prey or near the optimum. Once the best agent is determined, the other agents attempt to change their positions toward the best agent.

The optimization process of the WOA algorithm is summarized as follows:

Step 1. Set algorithm parameters.

Step 2. Initialize the whales' population $X_{i}(i=1,2, \ldots, n)$.

Step 3. Calculate the fitness of each search agent $f\left(\vec{X}_{i}\right)$.

Step 4. Update the parameters of the algorithm.

Step 5. Generate a random number $p$ in $[0,1]$. Select different strategies of location update according to $p$.

Step 6. Return to Step 3 until optimal values $r$ and $\gamma$ are determined.

\section{Validation of the CFNGBM $(1,1)$}

We present two cases to verify the efficacy of the proposed model compared with five competing models, including the traditional FANGM [28], FAGM [13], NGMO [29], NGM [11], and FANGBM [21]. Moreover, to evaluate the forecasting accuracy of these grey models, we applied certain statistical indicators to assess the quality of the model, which are defined as follows:

mean absolute percentage error: MAPE

$$
=\left(\frac{1}{n}\right) \sum_{k=1}^{n}\left|\frac{x^{(0)}(k)-\hat{x}^{(0)}(k)}{x^{(0)}(k)}\right| \times 100,
$$

mean absolute: $\operatorname{MAE}=\left(\frac{1}{n}\right) \sum_{k=1}^{n}\left|\widehat{x}^{(0)}(k)-x^{(0)}(k)\right|$,

mean squares error: $\mathrm{MSE}=\frac{1}{n} \sum_{k=1}^{n}\left(\widehat{x}^{(0)}(k)-x^{(0)}(k)\right)^{2}$,

root mean squares percentage error: RMSPE

$$
=\sqrt{\left(\frac{1}{n}\right) \sum_{k=1}^{n}\left(\frac{\hat{x}^{(0)}(k)-x^{(0)}(k)}{x(0)(k)}\right)^{2}} \times 100,
$$

correlation coefficient: $R=\frac{\operatorname{Cov}\left(\widehat{\mathbf{x}}^{(0)}, \mathbf{x}^{(0)}\right)}{\sqrt{\operatorname{Var}\left[\widehat{\mathbf{x}}^{(0)}\right] \operatorname{Var}\left[\mathbf{x}^{(0)}\right]}}$

Case 1. Forecasting of $\mathrm{CO}_{2}$ emissions in China 
TABLE 2: The minimum MAPE and optimal order, power index of $\operatorname{FAGM}(1,1), \operatorname{FANGM}(1,1, k, c), \operatorname{FNGBM}(1,1)$, and CFNGBM( $(1,1)$, and $\mathrm{CO}_{2}$ emissions in China.

\begin{tabular}{cccccccccc}
\hline & \multicolumn{2}{c}{ CFNGBM } & \multicolumn{3}{c}{ FNGBM } & \multicolumn{2}{c}{ FAGM } & \multicolumn{2}{c}{ FANGM } \\
$r$ & $\gamma$ & MAPE (\%) & $r$ & $\gamma$ & MAPE (\%) & $r$ & MAPE (\%) & $r$ & MAPE (\%) \\
\hline 0.951 & 0.520 & $\mathbf{2 . 4 4 6}$ & 0.23 & 0.59 & 2.87 & 0.268 & 4.9512 & 0.8681 & 3.7164 \\
\hline
\end{tabular}

TABLe 3: The minimum MAPE and time consumption of WOA, PSO, and GA in Case 1.

\begin{tabular}{cccccc}
\hline & WOA & PSO & GA & \\
MAPE $(\%)$ & Time consumption(s) & MAPE $(\%)$ & Time consumption(s) & MAPE(\%) & Time consumption(s) \\
\hline 2.446 & $\mathbf{6 . 2 1 1}$ & $\mathbf{2 . 0 4 3 1}$ & 62.618 & 2.1405 & 16.386 \\
\hline
\end{tabular}

TABLE 4: Results of $\mathrm{CO}_{2}$ emissions in China using six models.

\begin{tabular}{|c|c|c|c|c|c|c|c|}
\hline Year & Raw data & FANGM & FAGM & NGMO & NGM & CFNGBM & FNGBM \\
\hline 2000 & 3362.7000 & 3362.700 & 3362.700 & 3362.700 & 3362.700 & 3362.700 & 3362.700 \\
\hline 2001 & 3525.0000 & 3292.203 & 3737.913 & 3266.673 & 2734.660 & 2924.815 & 3630.167 \\
\hline 2002 & 3845.4000 & 4046.790 & 4313.449 & 4050.256 & 3565.453 & 3781.706 & 4196.168 \\
\hline 2003 & 4534.4000 & 4758.969 & 4882.322 & 4763.443 & 3565.453 & 4570.822 & 4807.091 \\
\hline 2004 & 5337.0000 & 5411.520 & 5421.771 & 5412.557 & 5009.980 & 5291.335 & 5411.459 \\
\hline 2005 & 6099.5000 & 6005.557 & 5929.415 & 6003.355 & 5636.505 & 5944.246 & 5988.919 \\
\hline 2006 & 6677.9000 & 6545.085 & 6406.808 & 6541.077 & 6206.782 & 6531.700 & 6529.440 \\
\hline 2007 & 7240.3000 & 7034.704 & 6856.271 & 7030.490 & 6725.863 & 7056.520 & 7027.942 \\
\hline 2008 & 7378.5000 & 7479.004 & 7280.102 & 7475.935 & 7198.342 & 7521.915 & 7482.242 \\
\hline 2009 & 7708.8000 & 7882.348 & 7680.392 & 7881.361 & 7628.404 & 7931.286 & 7892.007 \\
\hline 2010 & 8135.2000 & 8248.796 & 8058.993 & 8250.365 & 8019.857 & 8288.104 & 8258.135 \\
\hline 2011 & 8805.8000 & 8582.073 & 8417.533 & 8586.217 & 8376.1678 & 8595.829 & 8582.339 \\
\hline 2012 & 8991.5000 & 8885.570 & 8757.449 & 8891.896 & 8700.489 & 8857.853 & 8866.861 \\
\hline 2013 & 9237.7000 & 9162.358 & 9080.014 & 9170.114 & 8995.695 & 9077.469 & 9114.256 \\
\hline 2014 & 9223.7000 & 9415.204 & 9386.364 & 9423.337 & 9264.398 & 9257.840 & 9327.248 \\
\hline 2015 & 9174.6000 & 9646.596 & 9677.513 & 9653.810 & 9508.978 & 9401.992 & 9508.613 \\
\hline 2016 & 9119.0000 & 9858.765 & 9954.379 & 9863.578 & 9731.601 & 9512.802 & 9661.103 \\
\hline 2017 & 9229.8000 & 10053.71 & 10217.79 & 10054.50 & 9934.2376 & 9592.993 & 9787.389 \\
\hline 2018 & 9428.7000 & 10233.21 & 10468.51 & 10228.27 & 10118.68 & 9645.137 & 9890.026 \\
\hline
\end{tabular}

With China's rapid economic advancement, the use of energy has considerably increased, leading to a significant increase in $\mathrm{CO}_{2}$ emissions. Increased $\mathrm{CO}_{2}$ emission warms the climate and causes natural disasters, such as melting glaciers. Effective $\mathrm{CO}_{2}$ emissions forecasting can enable early warning and help decision-makers to devise rational plans. Here, data from 2000 to 2014 were used to fit the model and data from 2015 to 2018 were used to validate the model (source: BP Statistical Review of World Energy 2019). To optimize the parameters of the CFNGBM model with all data, we used the WOA. From Table 2, we observe that the CFNGBM's order $r$ and power index $\gamma$ are 0.951 and 0.520 , respectively; its MAPE is 2.446, which is the smallest of the four models (the results of FAGM and FANGM are obtained from the work of $\mathrm{Wu}$ et al. [22].)

In this case, to verify the advantages of WOA in CFNGBM, the classic optimization algorithms including PSO and GA are selected as benchmarks. Table 3 shows that the MAPE of WOA, PSO, and GA is $2.446 \%, 2.043 \%$, and $2.1405 \%$, respectively, and computation times are taken as $6.211 \mathrm{~s}, 62.618 \mathrm{~s}$, and $16.386 \mathrm{~s}$, respectively, which indicates that although the accuracy of WOA is not the best (close to the best), the time taken by WOA is significantly lower than that of other competitors.

The obtained parameters are used to fabricate a model and make predictions. Table 4 shows the results of the simulation and prediction of $\mathrm{CO}_{2}$ emissions in China using six models.

To verify the accuracy of each model, equations (28)-(32) were used. We can observe from Table 5 that during the simulation phase, the MAE, MSE, MAPE, RMSPE, and $R$ of the CFNGBM are 152.5, 41945, 2.6866, 4.749 , and 0.9961 , respectively. Although the indicators of CFNGBM are not the best, they are close to the best. In the forecast stage, the MAE, MSE, MAPE, and RMSPE of the CFNGBM are $300.2,96338,3.256,3.374$, and 0.737 , respectively, thus achieving the best results among all models.

Case 2. Forecasting of $\mathrm{CO}_{2}$ emissions in India

India is the second-most populous country in the world. The utility of energy increases with environmental pressure. In particular, the increase in $\mathrm{CO}_{2}$ emissions causes many 
TABLE 5: Error metrics of $\mathrm{CO}_{2}$ emissions in China.

\begin{tabular}{|c|c|c|c|c|c|c|}
\hline Fitting & FANGM & FAGM & NGMO & NGM & CFNGBM & FNGBM \\
\hline MAE & 153.5 & 220.3 & 155.8 & 316.9881 & 152.5 & 150.6 \\
\hline MSE & 26872.8 & 65406.3 & 0.8231 & 138420.3 & 41945 & 29616 \\
\hline MAPE & 2.5842 & 3.7676 & 2.6513 & 5.5964 & 2.6866 & 2.54 \\
\hline RMSPE & 3.0 & 4.8804 & 3.2305 & 7.6696 & 4.749 & 3.35 \\
\hline $\mathrm{R}$ & 0.99 & 0.9948 & 0.9968 & 0.9953 & 0.9961 & 0.997 \\
\hline Prediction & FANGM & FAGM & NGMO & NGM & CFNGBM & FNGBM \\
\hline MAE & 710.0 & 841.5226 & 712.014 & 585.3498 & 300.2 & 473.7 \\
\hline MSE & 524020.8 & 752026.2 & 525870.6 & 364849.21 & 96383 & 232290 \\
\hline MAPE & 7.6790 & 9.09 & 7.7009 & 6.3281 & 3.256 & 5.12 \\
\hline RMSPE & 7.8225 & 9.356 & 7.8375 & 6.5233 & 3.374 & 5.22 \\
\hline $\mathrm{R}$ & 0.8144 & 0.823 & 0.8117 & 0.8117 & 0.737 & 0.784 \\
\hline
\end{tabular}

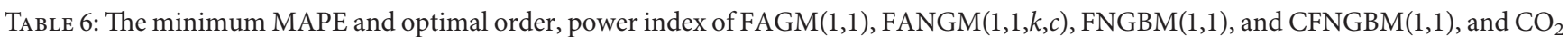
emissions in India.

\begin{tabular}{cccccccccc}
\hline & CFNGBM & & \multicolumn{3}{c}{ FANGBM } & \multicolumn{2}{c}{ FAGM } & \multicolumn{2}{c}{ FANGM } \\
$r$ & $\gamma$ & MAPE (\%) & $r$ & $\gamma$ & MAPE (\%) & $r$ & MAPE (\%) & $r$ & MAPE (\%) \\
\hline 1.1519 & 0.3081 & $\mathbf{1 . 0 7 4}$ & 0.129 & 0.03 & 1.148 & 0.1649 & 1.7519 & 1.2269 & 1.3275 \\
\hline
\end{tabular}

TABLE 7: The minimum MAPE and time consumption of WOA, PSO, and GA in Case 2.

\begin{tabular}{cccccc}
\hline & WOA & PSO & & GA \\
MAPE(\%) & Time consumption(s) & MAPE(\%) & Time consumption(s) & MAPE(\%) & Time consumption(s) \\
\hline 1.074 & $\mathbf{6 . 0 0 2 7}$ & 0.8559 & 58.882 & $\mathbf{0 . 8 4 8 8}$ & 17.598 \\
\hline
\end{tabular}

TABLE 8: Results of $\mathrm{CO}_{2}$ emissions in India using six models.

\begin{tabular}{|c|c|c|c|c|c|c|c|}
\hline Year & Raw data & FANGM & FAGM & NGMO & NGM & CFNGBM & FANGBM \\
\hline 2000 & 962.500 & 962.500 & 962.500 & 962.500 & 962.500 & 962.500 & 962.500 \\
\hline 2001 & 970.3000 & 980.592 & 933.755 & 946.740 & 942.075 & 967.941 & 946.603 \\
\hline 2002 & 1021.900 & 999.9330 & 979.664 & 1008.260 & 1003.321 & 989.666 & 990.837 \\
\hline 2003 & 1062.300 & 1055.790 & 1045.513 & 1073.200 & 1067.978 & 1049.63 & 1052.89 \\
\hline 2004 & 1116.600 & 1055.790 & 1120.686 & 1141.770 & 1136.236 & 1125.931 & 1124.093 \\
\hline 2005 & 1204.600 & 1202.766 & 1201.597 & 1214.160 & 1208.296 & 1208.185 & 1201.427 \\
\hline 2006 & 1252.500 & 1285.234 & 1286.709 & 1290.580 & 1284.370 & 1293.661 & 1283.573 \\
\hline 2007 & 1365.500 & 1371.716 & 1375.272 & 1371.260 & 1364.680 & 1381.476 & 1369.885 \\
\hline 2008 & 1466.900 & 1461.625 & 1466.901 & 1456.430 & 1449.464 & 1471.344 & 1460.032 \\
\hline 2009 & 1595.600 & 1554.655 & 1561.393 & 1546.340 & 1538.971 & 1563.200 & 1553.849 \\
\hline 2010 & 1661.000 & 1650.652 & 1658.650 & 1641.270 & 1633.462 & 1657.074 & 1651.268 \\
\hline 2011 & 1735.700 & 1749.547 & 1758.634 & 1741.490 & 1733.217 & 1753.031 & 1752.279 \\
\hline 2012 & 1849.200 & 1851.329 & 1758.634 & 1847.280 & 1838.527 & 1851.151 & 1856.909 \\
\hline 2013 & 1930.000 & 1956.018 & 1966.813 & 1958.980 & 1949.704 & 1951.521 & 1965.214 \\
\hline 2014 & 2083.300 & 2063.660 & 2075.077 & 2076.890 & 2067.072 & 2054.228 & 2077.268 \\
\hline 2015 & 2147.800 & 2174.317 & 2186.197 & 2201.370 & 2067.072 & 2159.356 & 2193.161 \\
\hline 2016 & 2234.200 & 2288.064 & 2300.237 & 2332.790 & 2321.785 & 2266.991 & 2312.993 \\
\hline 2017 & 2316.900 & 2404.985 & 2417.272 & 2471.540 & 2459.878 & 2377.216 & 2436.875 \\
\hline 2018 & 2479.100 & 2525.170 & 2537.379 & 2471.540 & 2605.662 & 2490.109 & 2564.927 \\
\hline
\end{tabular}

adverse changes. Thus, a reasonable prediction of $\mathrm{CO}_{2}$ emissions is significant for the country to formulate sustainable development strategies. In this case, we used all data to search the parameter order and power exponent. The results are shown in Table 6. CFNGBM's order $r$ and power index $\gamma$ are 1.1519 and 0.3081 , respectively; its MAPE is 1.074. (The results of FAGM and FANGM are obtained from the work of $\mathrm{Wu}$ et al. [22]).
Table 7 shows that the MAPE of WOA, PSO, and GA is $1.074 \%, 0.8559 \%$, and $\mathbf{0 . 8 4 8} \%$, respectively, and corresponding computation times are $\mathbf{6 . 0 0 2 7} \mathrm{s}, 58.882 \mathrm{~s}$, and $\mathbf{0 . 8 4 8 8}$ s, respectively. Similar to Case1, WOA still has a short running time in comparison with PSO and GA.

The obtained optimized parameters are used to establish a model and make predictions. Table 8 shows the results of 
TABLE 9: Error metrics of $\mathrm{CO}_{2}$ emissions in China.

\begin{tabular}{|c|c|c|c|c|c|c|}
\hline Fitting & FANGM & FAGM & NGMO & NGM & CFNGBM & FANGBM \\
\hline MAE & 14.7581 & 18.8077 & 17.8016 & 18.5138 & 15.190 & 15.611 \\
\hline MSE & 349.5036 & 4826.52 & 493.6639 & 13950.48 & 396.77 & 410.78 \\
\hline MAPE & 1.0421 & 1.4362 & 1.3239 & 4.7964 & 1.0909 & 1.150 \\
\hline RMSPE & 1.0421 & 1.9368 & 3.2305 & 1.6429 & 1.4800 & 1.520 \\
\hline $\mathrm{R}$ & 0.9987 & 0.9948 & 0.9982 & 0.9982 & 0.9985 & 0.9986 \\
\hline Prediction & FANGM & FAGM & NGMO & NGM & CFNGBM & FANGBM \\
\hline MAE & 53.6342 & 65.7712 & 712.0148 & 111.4319 & 28.918 & 82.48 \\
\hline MSE & 3371.48 & 4826.5 & 13950.48 & 11499.00 & 1242 & 7506 \\
\hline MAPE & 2.3264 & 2.8566 & 4.7964 & 4.3017 & 1.2633 & 3.569 \\
\hline RMSPE & 2.512 & 3.0094 & 5.0409 & 4.5704 & 1.5344 & 3.731 \\
\hline $\mathrm{R}$ & 0.9868 & 0.9867 & 0.9885 & 0.9885 & 0.9866 & 0.9872 \\
\hline
\end{tabular}

the simulation and prediction of $\mathrm{CO}_{2}$ emissions in India using six models.

We can observe from Table 9 that during the simulation phase, the MAE, MSE, MAPE, RMSPE, and $R$ of the CFNGBM model are 152.5, 41945, 2.6866, 4.749, and 0.9961, respectively. Although the indicators of CFNGBM are not the best, they are close to the best. In the forecast stage, the MAE, MSE, MAPE, and RMSPE of the CFNGBM are 300.2, $96338,3.256,3.374$, and 0.737 , respectively, achieving the best results among all models.

\section{Conclusion and Future Research}

The FANGBM, a successful generalization of the nonlinear grey Bernoulli model, generalizes the integer-order accumulation to a more general fractional-order accumulation and determines a more suitable accumulation order through intelligent optimization. Because of the optimization of order and power index, FANGBM has higher precision than some previous grey models. Although FANGBM $(1,1)$ has high accuracy and numerous excellent characteristics, its FA operator is relatively complicated to build model. Hence, it is difficult for general engineering technicians to master it. Herein, we propose the $\operatorname{CFNGBM}(1,1)$, wherein the fractional-order accumulation is implemented by CFA, which has a simpler structure. Using two practical examples of $\mathrm{CO}_{2}$ prediction in China and India, we determine that CFNGBM $(1,1)$ has the same accuracy (or higher prediction accuracy) as FANGBM $(1,1)$ and is a good substitute.

Although CFNGBM $(1,1)$ has many excellent characteristics, further work is required in the future. We list them as follows: (1) optimizing the background value of the CFNGBM $(1,1)$ model to achieve more high accuracy and (2) using more efficient intelligent optimization algorithms to further improve the modeling accuracy.

\section{Data Availability}

The data used to support the findings of this study are included in the article.

\section{Conflicts of Interest}

The authors declare that there are no conflicts of interest regarding the publication of this paper.

\section{Authors' Contributions}

Both the authors equally contributed to this work.

\section{Acknowledgments}

This work was supported by grants from the Postgraduate Research \& Practice Innovation Program of Jiangsu Province (grant no. KYCX20_1144).

\section{References}

[1] J.-L. Deng, "Control problems of grey systems," Systems \& Control Letters, vol. 1, no. 5, pp. 288-294, 1982.

[2] B. Zeng, H. Duan, Y. Bai, and W. Meng, "Forecasting the output of shale gas in China using an unbiased grey model and weakening buffer operator," Energy, vol. 151, pp. 238-249, 2018.

[3] B. Wei, N. Xie, and A. Hu, "Optimal solution for novel grey polynomial prediction model," Applied Mathematical Modelling, vol. 62, pp. 717-727, 2018.

[4] Z.-X. Wang and Q. Li, "Modelling the nonlinear relationship between $\mathrm{CO}_{2}$ emissions and economic growth using a PSO algorithm-based grey Verhulst model," Journal of Cleaner Production, vol. 207, pp. 214-224, 2018.

[5] X. Ma, M. Xie, W. Wu, B. Zeng, Y. Wang, and X. Wu, "The novel fractional discrete multivariate grey system model and its applications," Applied Mathematical Modelling, vol. 70, pp. 402-424, 2019.

[6] L. Wu, X. Gao, Y. Xiao, Y. Yang, and X. Chen, "Using a novel multi-variable grey model to forecast the electricity consumption of Shandong Province in China," Energy, vol. 157, pp. 327-335, 2018.

[7] L. Wu, N. Li, and Y. Yang, "Prediction of air quality indicators for the Beijing-Tianjin-Hebei region," Journal of Cleaner Production, vol. 196, pp. 682-687, 2018.

[8] Y. Wang, L. Pei, and Z. Wang, "The NLS-based grey Bass model for simulating new product diffusion," International Journal of Market Research, vol. 59, no. 5, pp. 655-669, 2017.

[9] X. Xiao, J. Yang, S. Mao, and J. Wen, “An improved seasonal rolling grey forecasting model using a cycle truncation accumulated generating operation for traffic flow," Applied Mathematical Modelling, vol. 51, pp. 386-404, 2017.

[10] N.-M. Xie and S.-F. Liu, "Discrete grey forecasting model and its optimization," Applied Mathematical Modelling, vol. 33, no. 2, pp. 1173-1186, 2009. 
[11] S.-F. Liu, "Novel grey forecasting model and its modeling mechanism," Control and Decision, vol. 24, pp. 1702-1706, 2009.

[12] B. Zeng and C. Li, "Forecasting the natural gas demand in China using a self-adapting intelligent grey model," Energy, vol. 112, pp. 810-825, 2016.

[13] L. Wu, S. Liu, L. Yao, S. Yan, and D. Liu, "Grey system model with the fractional order accumulation," Communications in Nonlinear Science and Numerical Simulation, vol. 18, no. 7, pp. 1775-1785, 2013.

[14] Y. Yang and D. Xue, "Continuous fractional-order grey model and electricity prediction research based on the observation error feedback," Energy, vol. 115, pp. 722-733, 2016.

[15] X. Zhu, Y. Dang, and S. Ding, "Using a self-adaptive grey fractional weighted model to forecast Jiangsu's electricity consumption in China," Energy, vol. 190, p. 116417, 2020.

[16] C.-I. Chen, H. L. Chen, and S.-P. Chen, "Forecasting of foreign exchange rates of Taiwan's major trading partners by novel nonlinear Grey Bernoulli model NGBM(1,1)," Communications in Nonlinear Science and Numerical Simulation, vol. 13, no. 6, pp. 1194-1204, 2008.

[17] J. Zhou, R. Fang, Y. Li, Y. Zhang, and B. Peng, "Parameter optimization of nonlinear grey Bernoulli model using particle swarm optimization," Applied Mathematics and Computation, vol. 207, no. 2, pp. 292-299, 2009.

[18] L.-C. Hsu, "A genetic algorithm based nonlinear grey Bernoulli model for output forecasting in integrated circuit industry," Expert Systems with Applications, vol. 37, no. 6, pp. 4318-4323, 2010.

[19] Z.-X. Wang, K. W. Hipel, Q. Wang, and S.-W. He, "An optimized $\operatorname{NGBM}(1,1)$ model for forecasting the qualified discharge rate of industrial wastewater in China," Applied Mathematical Modelling, vol. 35, no. 12, pp. 5524-5532, 2011.

[20] X. Liu and N. Xie, "A nonlinear grey forecasting model with double shape parameters and its application," Applied Mathematics and Computation, vol. 360, pp. 203-212, 2019.

[21] W. Wu, X. Ma, B. Zeng, Y. Wang, and W. Cai, "Forecasting short-term renewable energy consumption of China using a novel fractional nonlinear grey Bernoulli model," Renewable Energy, vol. 140, pp. 70-87, 2019.

[22] W.-Q. Wu, X. Ma, Y.-Y. Zhang, W.-P. Li, and Y. Wang, "A novel conformable fractional non-homogeneous grey model for forecasting carbon dioxide emissions of BRICS countries," Science of The Total Environment, vol. 707, 2020.

[23] H.-M. Duan and X.-L. Luo, "Grey optimization Verhulst model and its application in forecasting coal-related $\mathrm{CO}_{2}$ emissions," Environmental Science and Pollution Research, pp. 1-22, 2020.

[24] S. Ding, N. Xu, J. Ye, W.-J. Zhou, and X.-X. Zhang, "Estimating Chinese energy-related $\mathrm{CO}_{2}$ emissions by employing a novel discrete grey prediction model," Journal of Cleaner Production, vol. 259, 2020.

[25] X. Xin, W.-Q. Wu, B. Zeng, Y. Wang, and X.-X. Wu, "The conformable fractional grey system model," ISA Transactions, vol. 96, pp. 255-271, 2020.

[26] C.-I. Chen, P.-H. Hsin, and C.-S. Wu, "Forecasting Taiwan's major stock indices by the Nash nonlinear grey Bernoulli model," Expert Systems with Applications, vol. 37, no. 12, pp. 7557-7562, 2010.

[27] S. Mirjalili and A. Lewis, "The whale optimization algorithm," Advances in Engineering Software, vol. 95, pp. 51-67, 2016.

[28] W.-Q. Wu, X. Ma, B. Zeng, Y. Wang, and W. Cai, "Application of the novel fractional grey model FAGMO $(1,1, k)$ to predict China's nuclear energy consumption," Energy, vol. 165, pp. 223-234, 2018.

[29] P.-Y. Chen and H.-M. Yu, "Foundation settlement prediction based on a novel NGM model," Mathematical Problems in Engineering, vol. 2014, Article ID 242809, 8 pages, 2014. 